\title{
Experimental Study on The Impact of Antenna Characteristics on Non-stationary V2I Channel Parameters in Tunnels
}

\author{
Marwan Yusuf, Emmeric Tanghe, Pierre Laly, Frederic Challita, Bart Lannoo, Rreze Halili, \\ Rafael Berkvens, Maarten Weyn, Luc Martens, Davy P. Gaillot, Martine Lienard, and Wout Joseph
}

\begin{abstract}
This paper analyses the experimentally-assessed dual-polarized (DP) mobile channel in a tunnel environment at $1.35 \mathrm{GHz}$ under traffic conditions. We investigate the impact of antenna polarization and radiation pattern on the non-stationary vehicle-to-infrastructure (V2I) channel. Basic channel evaluation metrics are examined including path gain, co-polarization ratio (CPR), and cross-polarization discrimination (XPD). In addition, the stationarity region is estimated using the channel correlation function approach, and used to calculate the time-varying delay and Doppler power profiles. Statistical models are presented for parameters like CPR, XPD, RMS delay and Doppler spreads, where the lognormal distribution provides the best fit. The polarization and the opening angle of the antennas into the propagation channel are found to strongly influence the observed non-stationarity of the channel. They impact the degree of multipath richness that is captured, thus providing different path gain, delay and Doppler spreads. Based on our analysis, the directional antenna with vertical polarization provides the longest stationarity time of $400 \mathrm{~ms}$ at $90 \mathrm{~km} / \mathrm{h}$, as well as the highest path gain and lowest dispersion. Furthermore, the DP channel capacity is calculated and its dependence on different normalization approaches is investigated. We propose a more accurate normalization for the DP channels that takes the conservation of energy into account. Moreover, the subchannels correlation coefficients are determined. While the condition number is found to be low on average, the correlation results show high correlation among the DP subchannels. As conclusion, we show how the CPR and XPD play the main role in providing multiplexing gain for DP tunnel channels.
\end{abstract}

Index Terms-vehicular, propagation, measurement, delay, Doppler, correlation, tunnel, polarization, stationarity, MIMO normalization

\section{INTRODUCTION}

A $\mathrm{N}$ essential requirement for the development of vehicular communication systems is the accurate modeling of the propagation channel in different scenarios and environments [1]. One of the unexplored scenarios that needs more attention is tunnels. Being a confined environment, propagation behavior in tunnels differs from other environments as it plays the role of an oversized waveguide [2]. Deterministic channel

This research was supported by the VLAIO project "Smart Highway" and the EOS project "Multi-service Wireless Network (MUSE-WINET)".

Marwan Yusuf, Emmeric Tanghe, Luc Martens and Wout Joseph are with the Department of Information Technology, IMEC-WAVES, Ghent University, Belgium (e-mail: marwan.yusuf@ugent.be, emmeric.tanghe@ugent.be)

Frederic Challita, Pierre Laly, Davy P. Gaillot and Martine Lienard are with the Electronics Department, IEMN-TELICE, University of Lille, France.

Bart Lannoo, Rreze Halili, Rafael Berkvens, and Maarten Weyn are with IDLab - Faculty of Applied Engineering, University of Antwerp - imec.

Manuscript received XXX, XX, 2019; revised XXX, XX, 2019. models for tunnels include: waveguide models, ray tracing models and numerical methods for solving Maxwell's equations in tunnel environments [3]. These methods suffer from large computational complexity and incomplete description of the propagation environment (scatterers, mobility, traffic, etc.). On the other hand, analytical stochastic models that are obtained from measurements in real traffic conditions describe the specific environment with less computational cost [3]. As the propagation is influenced by many factors (e.g. tunnel geometry, obstacles, nodes setup, traffic), measurements in practical scenarios are required to characterize and model the propagation in tunnels.

Several studies have been published based on propagation measurements in tunnels. Some of these studies investigate propagation in subway tunnels [4], where the geometry and traffic conditions are different from road tunnels. Others investigate tunnels in terms of only path loss [5]. Authors in [6] study the field distribution in the transverse plane and the correlation in both transverse and longitudinal directions. These studies investigate propagation under no traffic conditions and do not include dispersion parameters like the delay spread. On the other hand, the work in [7] suggests that a singleslope model is more adequate for the path loss when there is traffic. Delay spread is measured in [8], [9] and compared to simulation results, but no statistical models are presented. In addition, the delay and Doppler spreads are evaluated in [10] for $\mathrm{V} 2 \mathrm{~V}$ in an empty tunnel, where a lognormal model is used to fit their statistical distribution. Similar results of the delay spread are reported in [11] for a small arched tunnel.

One of the main challenges for vehicular communications is the rapidly time-varying propagation channel. As a result, the widely used assumption of wide-sense stationarity (WSS) uncorrelated scattering (US) channel is no longer satisfied [12], [13]. For a non-stationary channel, the fading statistics change in time. Since communication algorithms often rely on the knowledge of second-order statistics of the channel, appropriate measures of the similarity between channel statistics are required, so that the fading parameters can be accurately evaluated and the channel modeling becomes physically meaningful. The non-stationary fading process of vehicular channels can be characterized by assuming local stationarity for a finite region in time and frequency. The author in [13] provides a theoretical framework that extends the scattering function of the WSSUS to a time-frequency (TF) dependent local scattering function (LSF). Based on the LSF, power 
profiles of the delay (PDP) and Doppler (DPP) can be estimated, and subsequent analysis of the corresponding second central moments can be performed. In addition, the channel correlation function (CCF), which describes the correlation of scatterers separated by lags in the time, frequency, delay and Doppler domains [13], can be used to estimate the stationarity time based on realistic measurements [14].

Multiple-input Multiple-output (MIMO) systems that exploit the polarization domain have recently gained increasing attention. Orthogonally polarized antennas often have high decorrelation; a major advantage for multiplexing systems. Making use of co-located dual-polarized (DP) antennas allows for compact antenna array design, which is essential for vehicular communications. The restrictions of equipment size, power, and cost can make it difficult, if not impossible, to physically mount the antennas of the vehicle far enough apart to achieve low correlation [15]. As the form factor of the antenna array becomes larger, this causes other challenges in engineering that may restrict MIMO technology (e.g. long cables or several distributed RF-chains). Moreover, such mounting choices are driven not only by performance and cost, but also by aesthetic design considerations [1]. Hence, DP antennas represent an attractive option for vehicular communications. However, when multi-polarized antennas are used, the choice of the polarization at the transmitter (Tx) and the receiver $(\mathrm{Rx})$ can result in substantially different propagation conditions. Depolarization mechanisms caused by scatterers and antenna design result in gain imbalance and correlation between channel matrix elements; a big disadvantage of DP MIMO. For an extensive literature overview of analytical and experimental studies related to DP channels, see [16]-[18] and references within. Nonetheless, very limited results on tunnels can be found [3].

It is thus important to explore the impact of antenna polarization on the propagation characteristics in tunnels. In this paper, we characterize a non-stationary V2I channel measured in a tunnel at $1.35 \mathrm{GHz}$ center frequency. We model the power gain $(\mathrm{G})$, co-polarization ratio (CPR), and cross-polarization discrimination (XPD). In addition to polarization, the impact of antenna radiation pattern is also explored by comparing the results of omni-directional and bi-directional antennas. It has been shown that directional antennas can potentially increase the mean duration of a connection by a factor of 4 when connecting from a vehicle to existing access points in suburban environment [20]. On the vehicular network level, the distribution of received frames over different angles of arrival in city-wide simulations shows a dominance of angles around $0^{\circ}$ and $180^{\circ}$ [21]. This indicates that most packets were received from vehicles in the front or in the back, that is, vehicles on the same street or even lane. Hence, bidirectional antennas provide a valid candidate for vehicular communications.

Moreover, we investigate the stationarity time of the DP channel based on the CCF introduced in [13], and statistically model the RMS delay and Doppler spreads in the channel across stationarity regions. Few studies have measured the stationarity time of DP channels [22], [23]; they use the empirical collinearity method in outdoor environments other than tunnels. The CCF method is compared to the collinearity method in [14] and is shown to have more advantages, e.g. not requiring a threshold. Finally, we investigate the MIMO capacity for DP channels, and give qualitative indications on the impact of different parameters like normalization, CPR, $\mathrm{XPD}$, and correlation coefficients. The contributions of this paper can be summarized as follows:

- Investigating the impact of antenna polarization and radiation pattern on the propagation characteristics in a tunnel environment under traffic conditions

- The channel power is modeled in terms of path gain, CPR and XPD

- Evaluating the stationarity time using the channel correlation function approach

- The RMS delay and Doppler spreads are statistically modeled across several regions of stationarity

- The DP capacity and correlation properties are calculated and compared to the kronecker statistical model

- An intuitive understanding of DP channel performance is presented by observing the impact of the power imbalance and normalization on the DP capacity

- A more accurate normalization approach of the DP MIMO matrix is proposed for capacity calculation based on the conservation of energy

The outline of the paper is as follows. The description of measurements and the methodology of analysis are presented in Section II. Section III describes the results and discussions, while conclusions are drawn in Section IV.

\section{Description of MeAsurements AND METHODOLOGY}

\section{A. Measurement Setup and Scenario}

Channel measurements are performed with the MIMOSA radio channel sounder [24]. It uses $80 \mathrm{MHz}$ of transmission bandwidth, centered around $1.35 \mathrm{GHz}$. This carrier frequency lies within the operating band of the LTE-V standard [19] radio interface that supports V2I communications (named Uu-interface), which operates in the licensed $2 \mathrm{GHz}$ band (880-2690 MHz). Identical sets of antennas are used at the Tx and Rx. For the omni-directional measurement (OM), a wideband antenna is used from Cobham Antenna Systems, model XPO2V-0.8-6.0/1441. It features vertical polarisation, $0.8-6 \mathrm{GHz}$ bandwidth, and $2 \mathrm{dBi}$ gain. For the bi-directional measurement (BM), two custom-made DP patch antennas of horizontal $(\mathrm{H})$ and vertical $(\mathrm{V})$ polarization are used back-toback, with one facing forward and the other facing backward. The peak gain is $7.4 \mathrm{dBi}$ and the half power beamwidth (HPBW) is $120^{\circ}$. The channel sounder is fully parallel; the data for all transmit antennas are simultaneously modulated onto the subcarriers using orthogonal frequency division multiplexing (OFDM), then allocated to the antenna elements in an interleaved scheme. Table I summarizes the technical configuration of the MIMOSA channel sounder used for this measurement campaign [24].

Measurements have been carried out in the Beveren tunnel in Antwerp, Belgium. The one-kilometer tunnel has two rectangular tubes of approximately $15 \mathrm{~m} \times 5 \mathrm{~m}$ cross-section, 


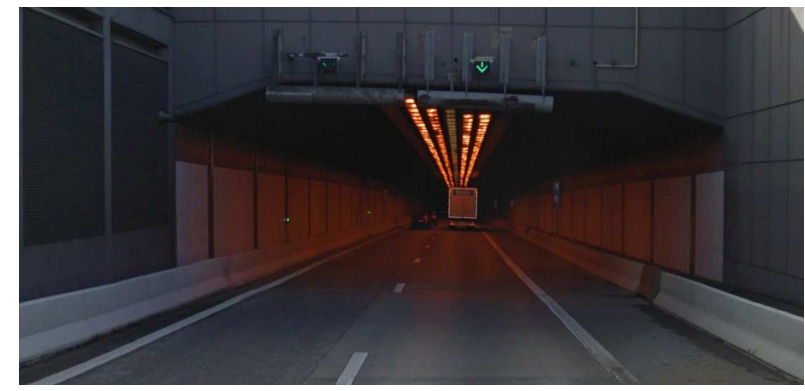

Fig. 1. Entrance of the Beveren Tunnel in Antwerp.

TABLE I

MIMOSA CHANNEL SOUNDER CONFIGURATION

\begin{tabular}{lr} 
Parameter & Setting \\
\hline Bandwidth & $80 \mathrm{MHz}$ \\
Center frequency & $1.35 \mathrm{GHz}$ \\
Tx and Rx polarization & $\mathrm{V}$ (omni), $\mathrm{V} / \mathrm{H}$ (patch) \\
Tx and Rx gain & $2 \mathrm{dBi}$ (omni), $7.4 \mathrm{dBi}$ (patch) \\
Tx and Rx HPBW (patch) & $120^{\circ}$ \\
OFDM symbol duration & $81.92 \mu \mathrm{s}$ \\
Minimum snapshot acquisition time & $327.68 \mu \mathrm{s}$ \\
Total recording time per trip & $33 \mathrm{~s}$ \\
\hline
\end{tabular}

with two lanes per direction, in addition to a roadside lane. Along the tunnel, there are lights, pipes and concrete blocks on the sides, as shown in Fig. 1. In order to follow the V2I scenario, the Tx antenna is placed inside around the middle of the tunnel through an emergency exit door at $2 \mathrm{~m}$ height, as shown in Fig. 2. The Rx antenna is mounted on the rooftop of a van carrying the $\mathrm{Rx}$ inside. The van is driven through the tunnel at $90 \mathrm{~km} / \mathrm{h}$ speed, crossing the Tx position during a measured trip of 33 seconds. The traffic condition was medium, with 10 to 15 vehicles present inside the tunnel during measurements. During the trip, the radio channel is captured with a snapshot repetition time $t_{\mathrm{s}}=3.92 \mathrm{~ms}$. With this parameters setting, we capture a total number of $S=8256$ snapshots per DP subchannel (VV, HV, VH, and HH). Each snapshot has $Q=819$ samples in the frequency domain. We achieve a maximum Doppler shift of $1 / 2 t_{\mathrm{s}}=128 \mathrm{~Hz}$ and a minimum resolvable delay resolution of $12.5 \mathrm{~ns}$.

\section{B. Methodology of analysis}

Due to the high mobility in vehicular communications, the environment is rapidly changing, and the observed fading process is non-stationary. The channel sounder provides a sampled measurement of the continuous channel transfer function (CTF) that is time-varying and frequency selective $h(t, f)$. We start our analysis by characterizing the path gain inside the tunnel, along with the CPR and XPD. Then, the stationarity time and delay-Doppler dispersion are analyzed. We compare these parameters for different polarizations and antenna patterns at $\mathrm{Tx}$ and Rx. Finally, we investigate the DP $2 \times 2$ MIMO channel in terms of capacity and related parameters, including the condition number and correlation matrix.

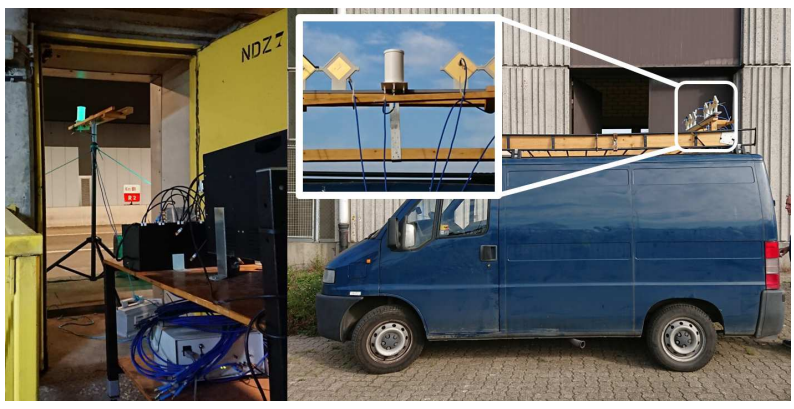

Fig. 2. Tx as a roadside unit with the antenna fixed inside the tunnel through an emergency exit (left) and the van loaded with Rx and the antenna mounted on the rooftop (right).

1) Power Versus Distance: We calculate the channel path gain by averaging the power gain over the 819 frequency subcarriers for each polarization as a function of distance

$$
G_{i j}=\mathrm{E}\left\{\left|h_{i j}\right|^{2}\right\}
$$

where $i, j \in\{\mathrm{V}, \mathrm{H}\}$ is the polarization at $\mathrm{Tx}$ and $\mathrm{Rx}$, respectively. The CPR is the power ratio between the two copolarized subchannels gain, given by the following formula in $\mathrm{dB}$

$$
\mathrm{CPR}=10 \log _{10}\left(\frac{G_{\mathrm{VV}}}{G_{\mathrm{HH}}}\right) .
$$

The XPD is the power ratio between a co-polarized subchannel gain and the corresponding cross-polarized subchannel gain. It shows the amount of depolarization or power leakage that each of the $\mathrm{H}$ and $\mathrm{V}$ subchannel goes through. The following formula represents XPD in $\mathrm{dB}$

$$
\begin{aligned}
& \mathrm{XPD}_{\mathrm{V}}=10 \log _{10}\left(\frac{G_{\mathrm{VV}}}{G_{\mathrm{VH}}}\right), \\
& \mathrm{XPD}_{\mathrm{H}}=10 \log _{10}\left(\frac{G_{\mathrm{HH}}}{G_{\mathrm{HV}}}\right) .
\end{aligned}
$$

According to [7], a one-slope model can be used to fit the path loss in tunnels with road traffic conditions. Hence, we use the following log-distance path loss model

$$
G(d)=G_{0}-n 10 \log _{10}(d)+X_{\sigma},
$$

where $G(d)$ is the path gain in $\mathrm{dB}$ at distance $d$ from Tx, $G_{0}$ is the reference value at $1 \mathrm{~m}, n$ is the path loss exponent, and $X_{\sigma}$ is a random variable with normal distribution of zero mean and $\sigma$ standard deviation. These parameters are determined by a least-squares fit to the measurement data.

2) LSF Estimate: The LSF is a TF-dependent representation of the power spectrum of the observed fading process in the delay and Doppler domains $(\tau, v)$. We use the discrete version of the LSF multitaper-based estimator proposed in [13]. The 2-D tapering windows applied to the CTF are computed from the discrete prolate spheroidal sequences and the number of used tapers is 2 in both time and frequency domains. We estimate the LSF for consecutive regions in time, within which the channel is assumed to be WSSUS. A sliding window is used with an extent of $M \times N$ samples in time and frequency domains, respectively. Further details can be found 


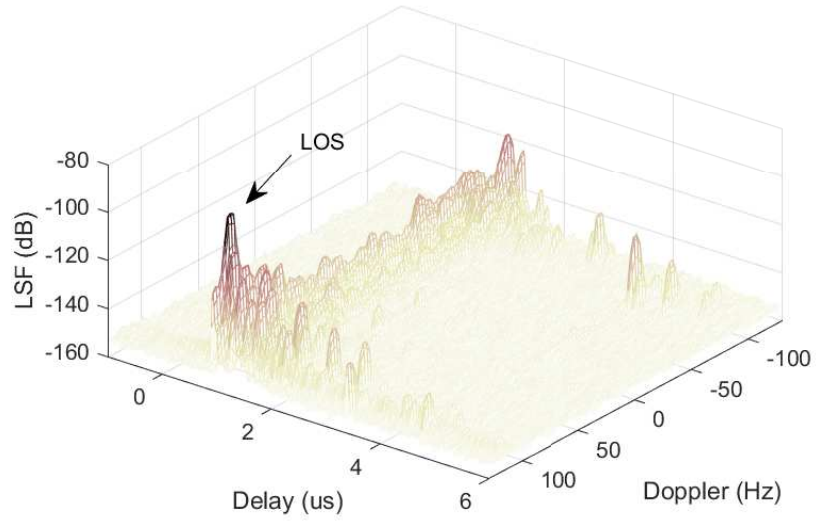

(a)

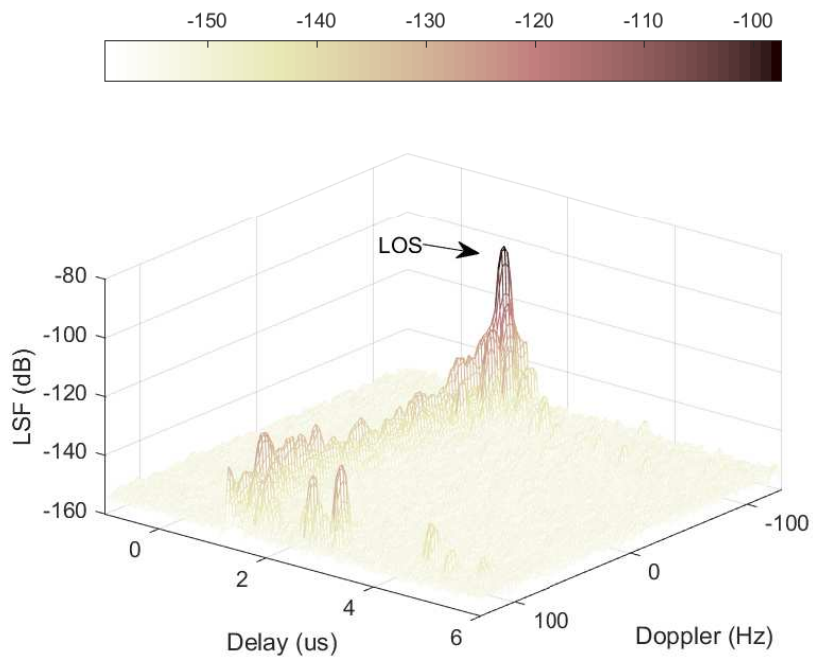

(b)

Fig. 3. LSF estimates at two instants before (a) and after (b) crossing the Tx position.

in [14]. Fig. 3 shows two LSF estimates at different instants during the trip: (a) when $\mathrm{Rx}$ is approaching $\mathrm{Tx}$, which can be seen from the positive Doppler shift of the LOS component around $100 \mathrm{~Hz}$, and (b) after Rx crosses Tx position, resulting in the LOS Doppler shift from positive to negative $100 \mathrm{~Hz}$.

3) Stationarity Region: The stationarity region represents the region in time and frequency within which the LSF is highly correlated. For calculating the stationarity time, we first need to estimate the LSF assuming a minimum stationarity region. This initial region should be small enough not to include non-stationary variations, and large enough to include sufficient resolution in the Doppler domain. We choose the region's dimension in time domain $M=32$ samples, corresponding to $125 \mathrm{~ms}$ or $15 \lambda$, approximately. This needs to be validated after we calculate the stationarity time; that it is indeed larger than the initial value. Since we are interested in the stationarity time, we include the whole bandwidth of $N=Q=819$ samples in frequency domain. The sliding time shift is selected to be half of the window size, i.e. $62 \mathrm{~ms}$ in this case. With these parameters, we obtain a LSF estimate of $12.5 \mathrm{~ns}$ delay resolution and $8 \mathrm{~Hz}$ Doppler resolution.

The stationarity time can be calculated from the spread of the CCF about the origin in the Doppler lag direction [13]. We use a discrete implementation of the $\mathrm{CCF}$, considering only the Doppler lag $\Delta v$ dependency for calculating the stationarity time [14]. The CCF estimate $\hat{A}$ is the 3-D DTFT of the LSF estimate $\hat{L}$

$$
\hat{A}[\Delta v, \Delta f, \Delta t]=\mathbf{F}^{3}\{\hat{L}[t, \tau, v]\},
$$

where $\Delta t, \Delta f$ and $\Delta v$ are the lag indexes in time, frequency and Doppler domains, respectively. Note that $(t, \Delta v),(\tau, \Delta f)$, and $(v, \Delta t)$ each are Fourier dual variables [13]. Then, we calculate the CCF Doppler moment as

$$
\hat{s}^{(\Delta v)}=\frac{1}{\|\hat{A}\|_{1}} \sum_{\Delta v} \sum_{\Delta t} \sum_{\Delta f}|\Delta v||\hat{A}[\Delta v, \Delta f, \Delta t]|,
$$

from which the stationarity time can be evaluated as [13]

$$
T_{\mathrm{s}}=\frac{1}{\hat{s}^{(\Delta v)}} .
$$

4) Delay and Doppler Spreads: The second-order central moments of the PDP and DPP are of great importance and relevance to fading channels characterization and systems design. They have been usually assumed constant for a certain environment. However, the non-stationarity of vehicular channels allows such parameters to be defined only within a local region of stationarity. Therefore, it is reasonable to characterize the delay and Doppler spreads as time-varying channel parameters. The PDP and DPP are the integral of the LSF estimate per stationarity region over the Doppler and delay domains, respectively.

Based on the estimated profiles, the time-varying RMS delay and Doppler spreads can be calculated. Pre-processing is carried out for each stationarity region separately before calculating the spreads. In order to avoid spurious and noise components, we only consider components within $40 \mathrm{~dB}$ from the peak level of the estimated LSF. All components below that power threshold are set to zero [10].

5) Channel Capacity: For a narrowband system of $n_{T}$ Tx antennas and $n_{R} \mathrm{Rx}$ antennas, the maximum capacity expressed in bits/s/Hz, with uniform power allocation and the presence of additional white Gaussian noise is given by the generalized formula

$$
C=\log _{2} \operatorname{det}\left(\mathbf{I}_{n_{R}}+\frac{\mathrm{SNR}}{n_{T}} \mathbf{H H}^{\dagger}\right),
$$

where $\mathbf{I}_{n_{R}}$ is the identity matrix of size $n_{R}$, SNR is the average signal-to-noise ratio per $\mathrm{Rx}$ antenna, $\mathbf{H}$ is the $n_{T} \times n_{R} \mathrm{CTF}$ matrix, and (. $)^{\dagger}$ is the Hermitian transpose. The wideband capacity is calculated by averaging $C$ over the frequency bandwidth.

In our scenario, the CTF matrix is expressed as

$$
\mathbf{H}=\left[\begin{array}{ll}
h_{\mathrm{VV}} & h_{\mathrm{VH}} \\
h_{\mathrm{HV}} & h_{\mathrm{HH}}
\end{array}\right] .
$$

Since the actual SNR varies as a function of $\mathrm{Rx}$ location, channel normalization is required to facilitate comparison of 
the results at a constant SNR. One common normalization is to scale the channel matrices such that the average power transfer between a single $\mathrm{Tx}$ and single Rx antenna is unity. The unity power gain constraint is equivalent to setting the squared Frobenius norm of the normalized matrix as $\mathrm{E}\left\{\|\mathbf{H}\|_{\mathrm{F}}^{2}\right\}=$ $n_{R} n_{T}$ [25]-[27].

6) Correlation Properties: Subchannels correlation is considered to have a strong relation to the performance of MIMO systems [28]. In order to characterize the correlation properties of the measured DP channel, the full correlation matrix is calculated using the following expression for its elements

$$
\rho_{i, j}=\frac{\mathrm{E}\left\{h_{i} h_{j}^{*}\right\}-\mathrm{E}\left\{h_{i}\right\} \mathrm{E}\left\{h_{j}^{*}\right\}}{\sqrt{\mathrm{E}\left\{\left|h_{i}\right|^{2}-\left|\mathrm{E}\left\{h_{i}\right\}\right|^{2}\right\} \mathrm{E}\left\{\left|h_{j}\right|^{2}-\left|\mathrm{E}\left\{h_{j}\right\}\right|^{2}\right\}}},
$$

where $i, j \in\{\mathrm{VV}, \mathrm{VH}, \mathrm{HV}, \mathrm{HH}\}$ are the subchannels under consideration. An alternative way to describe the channel correlation is to utilize the Kronecker model. In that model, the MIMO system is decomposed into two interconnected subsystems, with one having the correlation matrix at Tx side and the other at $\mathrm{Rx}$ side. The model approximates the correlation matrix as the Kronecker product of correlation matrices at $\mathrm{Tx}$ and $\mathrm{Rx}$ separately. It works well at large Tx$\mathrm{Rx}$ distances where the propagation at $\mathrm{Tx}$ and $\mathrm{Rx}$ can be considered independent [28], [29]. Since the Kronecker model is merely an approximation, the difference between the model and the measured results can be quantified using the second order error statistics given by [30]

$$
\epsilon=\frac{\left\|\mathbf{R}_{K}-\mathbf{R}\right\|_{\mathrm{F}}}{\|\mathbf{R}\|_{\mathrm{F}}}
$$

where $\mathbf{R}_{K}$ and $\mathbf{R}$ are the correlation matrices from the Kronecker model and measurements, respectively.

\section{RESUlTS AND Discussion}

\section{A. Path Gain, CPR and XPD}

The channel gain is calculated from the CTF by averaging the power gain over all frequencies per subchannel. The one-slope model in (5) is used to characterize the distance dependence of the path gain. Fig. 4 shows the measured channel gain versus distance during the second half of the tunnel, i.e. after Rx crosses Tx, and the corresponding model for different polarizations of the BM. Table II summarizes the model parameters, where the results of the OM model is also included for comparison.

A general observation is that the path loss exponent is smaller than in free-space and typical outdoor environments. This is due to the guiding effect of the tunnel, making it closer to indoor scenarios like industrial environments [31]. We also notice a periodic pattern in the fading over distance. In empty tunnels, the field fluctuations are mainly related to the richness in terms of propagating modes. According to the modal theory [32], the superposition of several hybrid modes supported by the structure of the tunnel is what gives rise to large pseudoperiods with distance on the large scale. However, the traffic condition disturbs the propagating modes, making the pseudoperiods in Fig. 4 less clear than in empty tunnels [33].

Regarding the polarization dependence, we see that the cross-polarized subchannels have lower reference gain than the

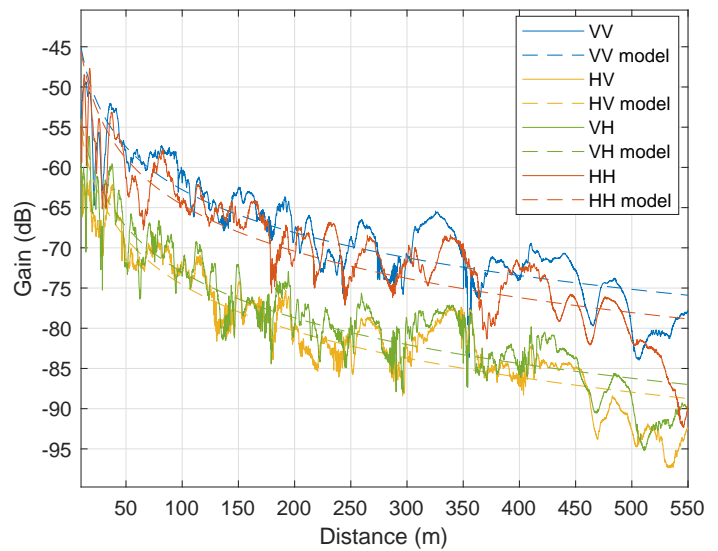

Fig. 4. Path gain versus distance for different polarization combinations.

TABLE II

PATH Gain MOdEL PARAMETERS

\begin{tabular}{c|ccccc} 
& OM & VV & HV & VH & HH \\
\hline $\mathrm{G}_{0}$ & -42.6 & -37.00 & -49.22 & -48.84 & -39.54 \\
$n$ & 1.19 & 1.24 & 1.23 & 1.20 & 1.27 \\
$\sigma$ & 3.47 & 4.66 & 4.12 & 4.30 & 3.38 \\
\hline
\end{tabular}

co-polarized subchannels, as expected. While the two crosspolarized models are almost identical, the co-polarized models show some differences: the $\mathrm{HH}$ subchannel has a slightly lower path gain than the VV channel. According to [34], a deterministic ray approach for empty smooth walled tunnels composed of uniform material predicts the opposite; the $\mathrm{H}$ path gain is higher than the V path gain. Since the geometry of the tunnel is such that the width is larger than the height, the $\mathrm{H}$ wave reflected from the ground and ceiling are stronger than the $\mathrm{V}$ one reflected from the walls of the tunnel, due to the Brewster's angle phenomenon [3], [35]. However, this effect can not be observed in the measurements. The reason is likely the non-uniformity of materials and shapes present in the propagation path (e.g. traffic condition, side pipes, trays and emergency exits), resulting in more scattering for the $\mathrm{HH}$ subchannel and a lower path gain.

We also investigate the effect of the antenna pattern by comparing the directional VV and OM models of same polarization. Due to the gain difference between the two antennas, the reference gain for the $\mathrm{OM}$ is $5.6 \mathrm{~dB}$ lower compared to the BM. In fact, the average gain within the HPBW of the $\mathrm{BM}$ is calculated as $5 \mathrm{dBi}$, and with a gain of $2 \mathrm{dBi}$ for the OM, the gain difference $=2 \times(5-2)=6 \mathrm{~dB}$. This is quite similar to the difference in the reference gain of the two models. This implies that, while the OM has a wider angle into the propagation environment, most of the multipath components with significant power arrive within the HPBW. Indeed, only rays impinging the tunnel walls with a grazing angle of incidence play a leading part in the propagation at large distance [33].

We further investigate the polarization dependence over distance in terms of CPR and XPR. Fig. 5 shows the CPR and XPD over the second half of the tunnel. It shows a slow 
TABLE IV

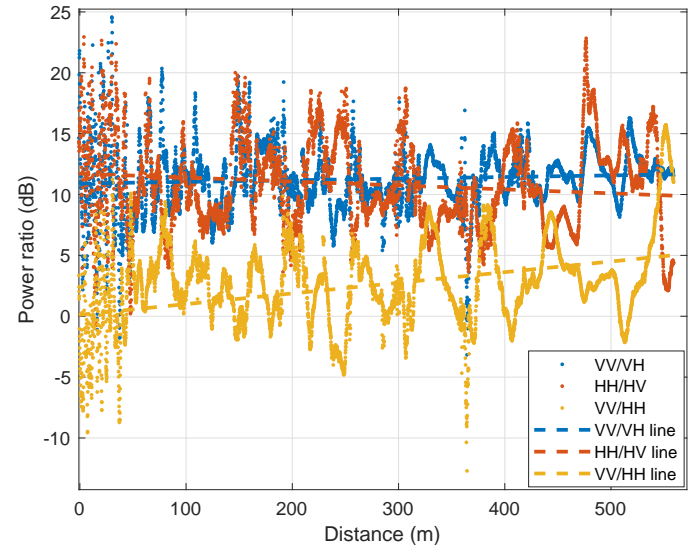

Fig. 5. CPR and XPD versus distance for different polarization combinations.

TABLE III

Statistical Model and Distance-DePendent Model Parameters OF CPR AND XPD

\begin{tabular}{c|cccc} 
& mean $(\mathrm{dB})$ & $\sigma(\mathrm{dB})$ & $R_{0}(\mathrm{~dB})$ & $R_{n}(\mathrm{~dB} / 100 \mathrm{~m})$ \\
\hline $\mathrm{CPR}$ & 3.53 & 3.57 & 0.35 & 1.0 \\
$\mathrm{XPD}_{\mathrm{V}}$ & 12.03 & 2.93 & 10.9 & 0.1 \\
$\mathrm{XPD}_{\mathrm{H}}$ & 11.29 & 3.49 & 10.6 & -0.6 \\
\hline
\end{tabular}

increase in the CPR with distance, which can be related to the small difference in the path loss exponent of VV and $\mathrm{HH}$ in Table II. On the other hand, the XPD does not drop at far distances and the waves remain highly polarized, confirming previous results [33], [36]. The ray theory of propagation in tunnels predicts that depolarization only happens at small range, where the waves impinging the tunnel walls are not polarized along the direction parallel or perpendicular to the plane of incidence [37].

Table III includes the mean values and the distance dependence parameters, where a linear dependence of $R_{0}+R_{n} d$ is assumed. In addition, the CPR and XPD are statistically modeled. Based on previous measurements and ray-tracing simulations, there is quite an agreement in the literature and standardized models that both parameters follow a lognormal distribution [17], [18], hence the estimated standard deviation is included in Table III. The results match with the values found in the literature for similar scenarios [18]. We notice that, while the instantaneous $\mathrm{XPD}_{\mathrm{V}}$ and $\mathrm{XPD}_{\mathrm{H}}$ are not identical, their models are similar, on average.

\section{B. Stationarity Region}

As aforementioned, the stationarity time represents the region in time within which the LSF is highly correlated. The LSF correlation can be determined by the CCF spread [13]. The CCF Doppler moment in (7) measures the CCF spread in the Doppler lag dimension, which is related to the stationarity time $T_{\mathrm{s}}$ in (8). It is worth noting that the CCF estimate from (6) may vary depending on the time interval of estimation. We choose to calculate it over the complete duration of the trip, thus, characterizing the degree of non-stationarity of the entire
STATIONARITY TIME STATISTICS IN MS FOR DIFFERENT RADIATION PATTERNS AND POLARIZATIONS

\begin{tabular}{c|ccccc}
$\mathrm{T}_{\mathrm{s}}(\mathrm{ms})$ & $\mathrm{OM}$ & $\mathrm{VV}$ & $\mathrm{HV}$ & $\mathrm{VH}$ & $\mathrm{HH}$ \\
\hline overall & $\mathbf{3 3 0}$ & $\mathbf{4 0 0}$ & $\mathbf{3 6 7}$ & $\mathbf{3 7 5}$ & $\mathbf{3 8 0}$ \\
mean & 428 & 435 & 432 & 427 & 436 \\
std. & 53 & 48 & 48 & 47 & 47 \\
min. & 268 & 325 & 315 & 299 & 303 \\
\hline
\end{tabular}

scenario in an average sense. This averages out effects due to temporary propagation conditions, which is more meaningful from an operational perspective since it is valid for the entire scenario.

Based on our measurement data of the overall trip in the tunnel, Table IV lists the estimated stationarity time in $\mathrm{ms}$ for each case. In addition, we add the mean, minimum and standard deviation statistics of the stationarity time calculated per $2 \mathrm{~s}$ period $(240 \lambda)$. It is clear that the stationarity time is indeed larger than the assumed minimum value of $125 \mathrm{~ms}$ in Section II-B for the LSF estimation. The results show that the co-polarized subchannels have longer stationarity times compared to the cross-polarized subchannels. This is expected as reported in [22], since the depolarized waves would undergo more variations due to reflection and scattering. We also notice that the $\mathrm{HH}$ subchannel has a shorter stationarity time than the VV subchannel. This can again be related to the conclusion that the $\mathrm{HH}$ subchannel experiences more scattering, and thus more time variation. The same conclusion can be drawn when comparing the OM with the VV subchannel; since the $\mathrm{OM}$ antenna has a wider angle of uniform gain, it captures more significant multipath components, contributing to a faster fading [38]. This results in the shortest stationarity time for the OM, compared to all subchannels of the BM.

\section{RMS Delay and Doppler Spreads}

For the non-stationary channel, the fading parameters can be accurately evaluated within each stationarity region, so that the channel modeling becomes physically meaningful. Based on our estimation of the stationarity time, the corresponding number of samples in the time domain is about $M=100$ samples. Hence, the LSF estimate is recomputed using a sliding window of the stationarity region dimensions. By integrating the LSF over the Doppler and delay domains, we get the PDP and DPP, respectively. Fig. 6 depicts the PDP and DPP for the VV subchannel. The LOS component can be easily recognized; the delay decreases as Rx approaches Tx with positive Doppler shift, then after crossing the Tx position around $11 \mathrm{~s}$, the delay starts increasing again and the Doppler shift becomes negative. Several multipath components can be observed; components from fixed scatterers are showing similar pattern to the LOS, i.e. Doppler shifts between $+/-100$ $\mathrm{Hz}$ as Rx passes by, but with less power. Components resulting from moving scatterers in the same movement direction have different Doppler shifts that are more consistent, depending on their relative speed and position.

The corresponding RMS delay and Doppler spreads [14] are shown in Fig. 7 for the VV and HH subchannels. The delay 


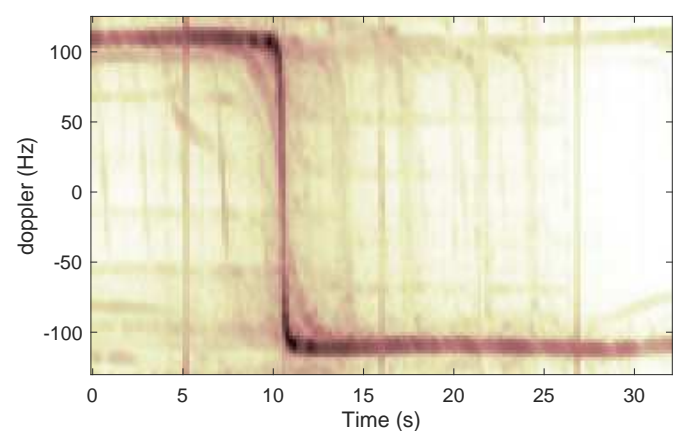

(a)

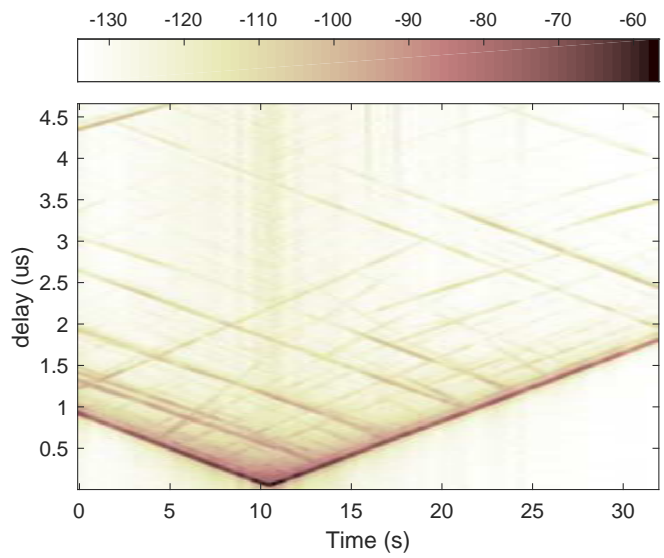

(b)

Fig. 6. Time-varying DPP (a) and PDP (b) for the VV subchannel in dB.

spread in the first half of the tunnel is larger compared to the second half. This can be related to the reflection coming from the metallic structure at the entrance of the tunnel, visible in Fig. 1. In addition, Fig. 7 shows that the spreads in the $\mathrm{HH}$ subchannel are relatively larger than in the VV subchannel. This confirms the previous conclusion, that the channel is more dispersive in the $\mathrm{H}$ polarisation, resulting in smaller path gain and stationarity time.

Previous studies find that a lognormal distribution is the best fit for the delay spread in tunnels [10], [11]. We verify this using the Lilliefors test [39]. It is a two-sided goodness-offit test when the parameters of the tested normal distribution are unknown and must be estimated, thus is suitable for our case. With 5\% significance level, the test shows that both the delay and Doppler spreads from the measurements follow the lognormal distribution. An example of the delay spread histogram for the $\mathrm{VV}$ and $\mathrm{HH}$ subchannels is shown in Fig. 8. Table $\mathrm{V}$ lists the estimated parameters of the lognormal distributions for different polarization combinations of the $\mathrm{BM}$, in addition to the $\mathrm{OM}$.

Looking at the BM results, the co-polarized subchannels have a larger delay spread but slightly smaller Doppler spread relative to the cross-polarized subchannels. Additionally, the $\mathrm{HH}$ subchannel has larger delay and Doppler spreads than the VV subchannel, as already mentioned. To show the impact of the antenna pattern, we compare the values of the OM and VV subchannel. The OM has larger delay and Doppler

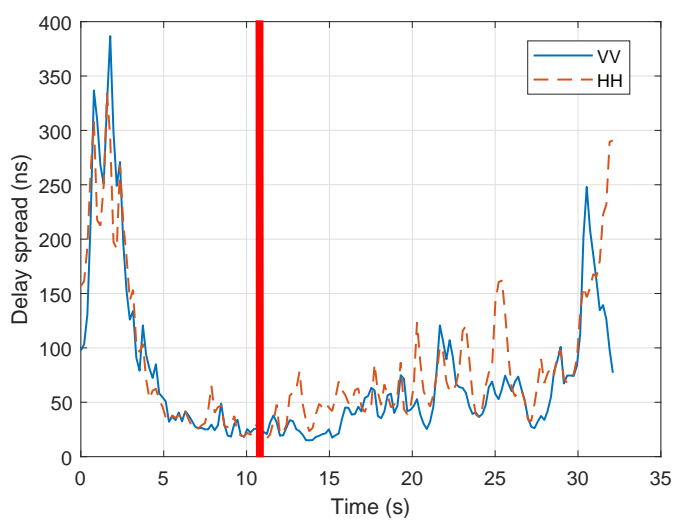

(a)

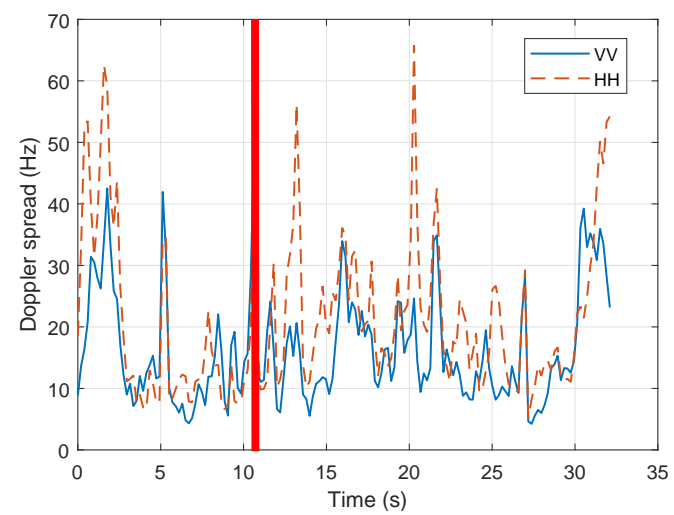

(b)

Fig. 7. RMS delay (a) and Doppler (b) spreads for VV and HH subchannels with Tx location in red.

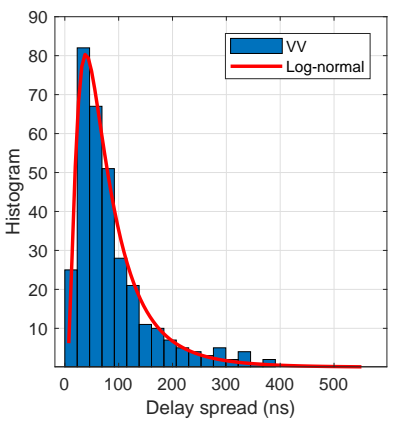

(a)

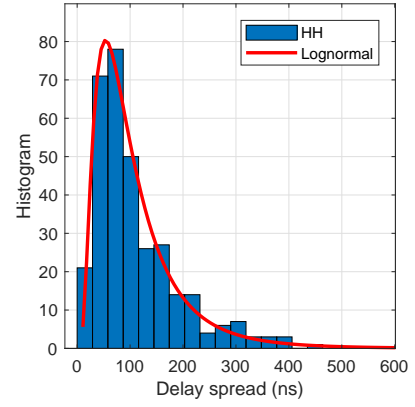

(b)
Fig. 8. Histograms of the RMS delay spread for the VV (a) and HH (b) subchannels and the corresponding lognormal models.

spreads, indicating that an antenna with wider angle captures more multipath components that increase dispersion. Hence, the impact of the antenna pattern on the spreads is larger than its impact on the power gain mentioned in Section IIIA. The reason is that the RMS spread includes the effect of both the power and the delay/Doppler of the multipath components, while only the average power is accounted for when calculating the gain. 
TABLE V

Statistics of The RMS Delay and Doppler SpReads Lognormal DISTRIBUTION

\begin{tabular}{c|ccc|ccc} 
& & Delay $(\mathrm{ns})$ & & & \multicolumn{3}{c}{ Doppler $(\mathrm{Hz})$} & \\
\cline { 5 - 6 } & mean & $\mu(\mathrm{dB})$ & $\sigma(\mathrm{dB})$ & mean & $\mu(\mathrm{dB})$ & $\sigma(\mathrm{dB})$ \\
\hline $\mathrm{OM}$ & 184.29 & 4.86 & 0.85 & 27.57 & 3.18 & 0.53 \\
$\mathrm{VV}$ & 87.96 & 4.2 & 0.74 & 18.88 & 2.73 & 0.63 \\
$\mathrm{HV}$ & 71.74 & 4.1 & 0.59 & 26.34 & 3.15 & 0.5 \\
$\mathrm{VH}$ & 72.1 & 4.1 & 0.63 & 23.28 & 3.02 & 0.5 \\
$\mathrm{HH}$ & 109.74 & 4.46 & 0.69 & 23.1 & 2.99 & 0.54 \\
\hline
\end{tabular}

\section{DP Channel Capacity and Normalization}

MIMO technology offers multiplexing and diversity without increasing the total system power and bandwidth, thus offering substantial improvements in channel capacity and spectral efficiency. DP MIMO has the benefit of reducing the antennas' form factor by having co-located DP antennas while maintaining low correlation, a condition usually required by MIMO systems [16]. However, in order to compare DP systems to other MIMO or even SISO systems, normalization is needed. The main idea is to isolate the small-scale characteristics of the channel from the effects of path loss and other largescale fading, so that the intrinsic characteristics of the MIMO matrix are compared at certain SNR. In this section, we will discuss three types of normalization. The goal is to investigate the effect of normalization on the accuracy of the DP channel capacity calculation, and propose a more accurate normalization approach.

1) Effect of normalization: The Frobenius normalization already mentioned in Section II-B would result in an average SISO SNR of unity on all the subchannels for a spatial array configuration. On the other hand, DP configurations suffer from power imbalances, which need to be accounted for in their capacity calculations. If the same normalization is used, the performance of these systems is overestimated [26], [27]. While some studies did use the Frobenius normalization for DP systems [23], [25], [36], others suggested normalizing the power of co-polarized subchannels [22], [26], or only one of the co-polarized subchannels (e.g. VV [40]) to unity.

To investigate the effect of normalization on the DP capacity, we calculate the capacity at $10 \mathrm{~dB}$ SNR using the different normalization approaches. Fig. 9 shows the DP capacity CDF when normalizing the CTF matrix to the power of the copolarized subchannels and the VV subchannel. The plots also include the SISO capacity of each subchannel, in addition to the DP capacity using the Frobenius normalization. We add the capacity of a $2 \times 2$ i.i.d. complex Gaussian MIMO channel as well for comparison. The theoretical average capacity at $10 \mathrm{~dB}$ SNR $=\log _{2}(1+10)=3.46 \mathrm{~b} / \mathrm{s} / \mathrm{Hz}$ for SISO, and $6.92 \mathrm{~b} / \mathrm{s} / \mathrm{Hz}$ for any $2 \times 2 \mathrm{MIMO}$ system. It is clear from the figures that the Frobenius normalization of the DP channel gives unrealistic results, as its capacity is larger than that of the i.i.d Gaussian channel. The effect of the power imbalance among the subchannels can be seen in the SISO capacity; the cross-polarized subchannels capacity is much lower than that of the co-polarized subchannels. That is because their SNR is lower than $10 \mathrm{~dB}$ due to the XPD. The effect of the

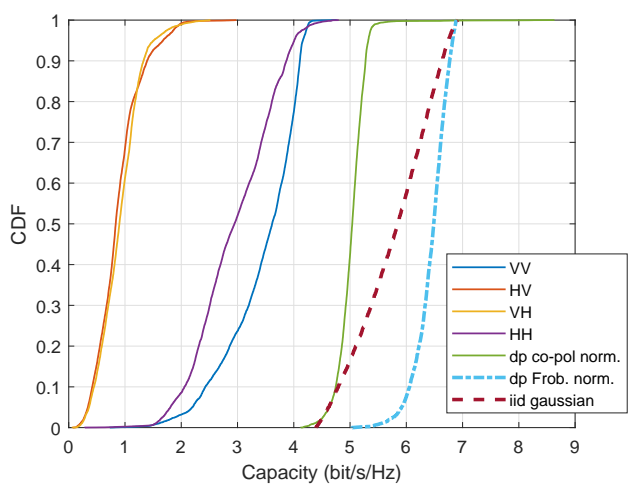

(a)

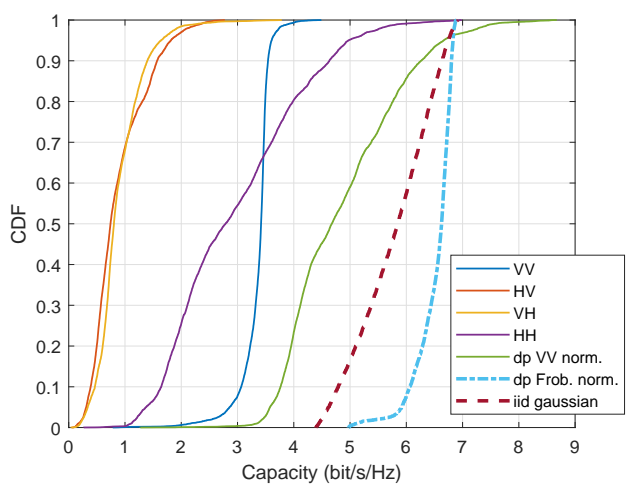

(b)

Fig. 9. CDF of the channel capacity when normalizing the power of the co-polarized subchannels (a) and the VV subchannel (b) to $10 \mathrm{~dB}$ SNR.

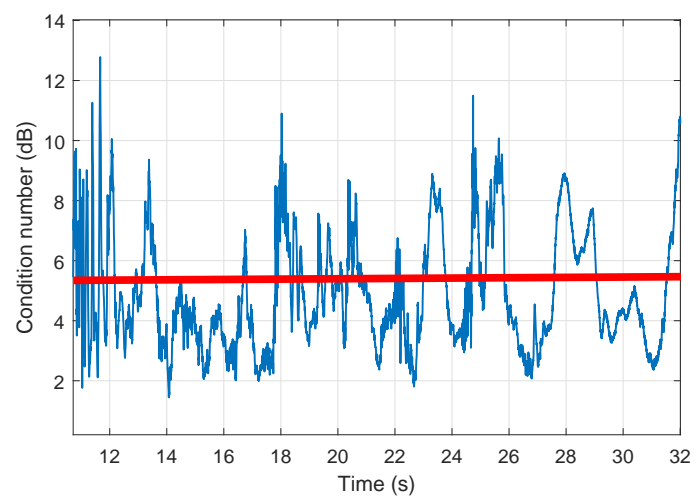

Fig. 10. The condition number during the second half of the tunnel, with the average in red.

CPR can be seen in the co-polarized subchannels capacity; in Fig. 9 (a) where the SNR is normalized to the co-polarized power, both $\mathrm{VV}$ and $\mathrm{HH}$ average capacities are around the theoretical value. Fig. 9 (b) shows the average VV capacity exactly at the theoretical value as expected, since its power is normalized. On the other hand, the $\mathrm{HH}$ capacity curve is more gradual, as its SNR deviates from the $10 \mathrm{~dB}$ of the VV subchannel due to the CPR.

Comparing the DP capacity of the two normalization approaches, we see that they do not overestimate the performance like the Frobenius normalization, as shown in Fig. 9. However, 


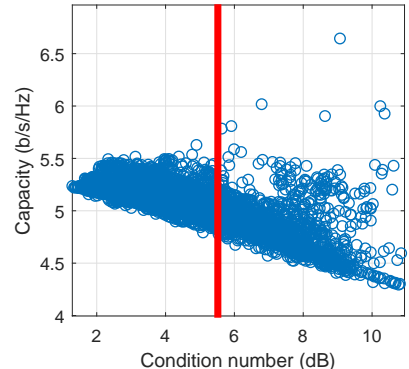

(a)

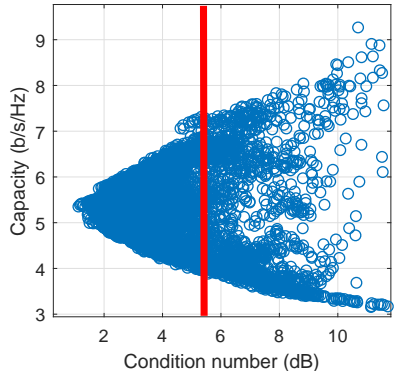

(b)
Fig. 11. Scatterplot of the condition number versus the DP capacity when normalizing the power of the co-polarized subchannels (a) and the VV subchannel (b) to $10 \mathrm{~dB}$ SNR, with the average condition number in red.

the power imbalance of the DP channel is still impacting the effective SNR, hence, the intrinsic MIMO matrix characteristics are not truly isolated. To show this, we need a parameter that describes the MIMO performance but does not depend on the channel normalization. The condition number is defined as the ratio of the maximum to minimum singular value of the channel matrix. The lower the condition number, the more potential the channel has for large multiplexing gains [27]. Fig. 10 shows the condition number of the CTF matrices during the second half of the tunnel. The average condition number is $5.5 \mathrm{~dB}$, which is good for having multiplexing gain in practice [41], [42]. We notice that the condition number remains relatively low as the SNR drops with distance. Fig. 11 shows the scatterplot of the condition number versus the capacity of the DP channel using the two normalization approaches. It is clear that the capacity is not correlated with only the condition number, indicating the impact of the power imbalance on the effective SNR.

2) Power conservation approach: The problem with these normalization approaches is that they do not assume a conservation of power or energy, where the channel cannot output more power than what is transmitted [16]. When normalizing to one or both of the co-polarized subchannels, the power imbalance due to CPR, and leakage from one polarization to the other due to XPD are not compensated for in the effective SNR. This makes the channel introduce more energy which is good for the performance, but unfortunately is unrealistic. Hence, we propose to normalize the channel matrix such that

$$
\mathrm{E}\left\{\|\tilde{\mathbf{H}}\|_{\mathrm{F}}^{2}\right\}=\frac{n_{R} n_{T}}{2} .
$$

In this normalization, the power is conserved by subtracting from the co-polarized subchannels SNR the corresponding amount of power that has leaked into the cross-polarized subchannels, i.e. we use $\mathrm{E}\left\{\left|h_{\mathrm{VV}}\right|^{2}\right\}+\mathrm{E}\left\{\left|h_{\mathrm{VH}}\right|^{2}\right\}=1$ and $\mathrm{E}\left\{\left|h_{\mathrm{HH}}\right|^{2}\right\}+\mathrm{E}\left\{\left|h_{\mathrm{HV}}\right|^{2}\right\}=1$ as constraints. Fig. 12 shows the capacity $\mathrm{CDF}$ and the scatterplot with the condition number using the proposed normalization. We notice that the capacity is fully correlated with the condition number, and has an average value of $4.82 \mathrm{~b} / \mathrm{s} / \mathrm{Hz}$. This implies that the proposed approach gives more accurate results, as it reflects the intrinsic MIMO gain, while insuring that the effective SNR is not overestimated.

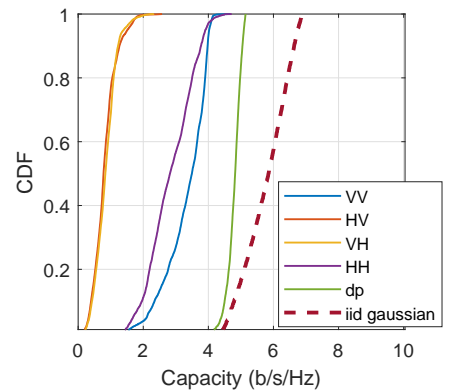

(a)

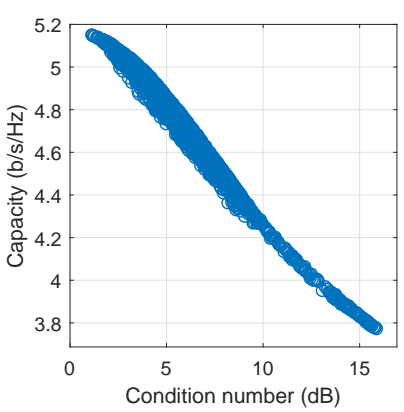

(b)
Fig. 12. CDF of the channel capacity (a) and its scatterplot versus the condition number (b) using the proposed normalization to $10 \mathrm{~dB}$ SNR.

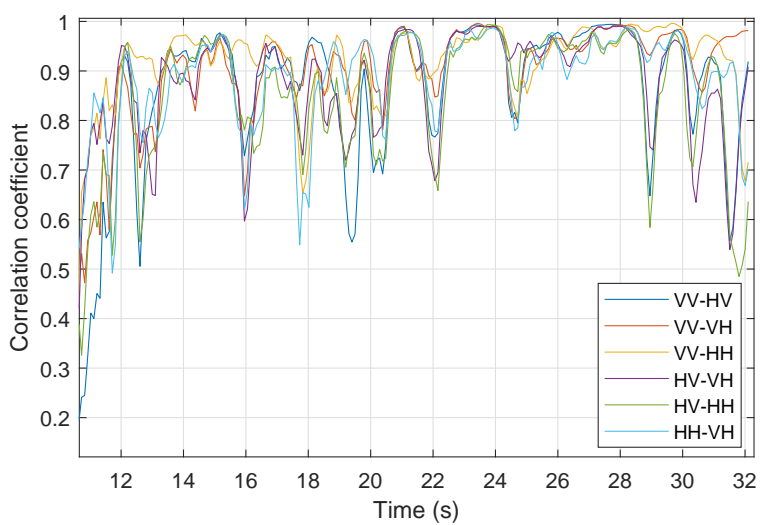

Fig. 13. Subchannels correlation coefficients during the second half of the tunnel.

\section{E. DP Subchannels Correlation}

Finally, we investigate the correlation among DP subchannels. In spatial MIMO, low correlation between antenna elements is often desired to enhance system capacity [16]. It was shown that DP waves in many NLOS scenarios fade almost independently, and they remain orthogonal throughout the channel in LOS scenarios [16]. We calculate the full correlation matrix whose elements are given in (11) as

$$
\mathbf{R}=\left[\begin{array}{llll}
1.00 & 0.86 & 0.87 & 0.90 \\
0.86 & 1.00 & 0.85 & 0.86 \\
0.87 & 0.86 & 1.00 & 0.87 \\
0.90 & 0.86 & 0.87 & 1.00
\end{array}\right]
$$

The results indicate high correlation values for the tunnel scenario. Similar results were observed for LOS scenarios in [23]. Alternatively, the Kronecker model can be used to describe the correlation properties, which is calculated as

$$
\mathbf{R}_{K}=\left[\begin{array}{llll}
1.00 & 0.86 & 0.86 & 0.75 \\
0.86 & 1.00 & 0.75 & 0.86 \\
0.86 & 0.75 & 1.00 & 0.87 \\
0.75 & 0.86 & 0.87 & 1.00
\end{array}\right]
$$

The kronecker model provides lower correlation values compared to the measurement results. The introduced error can be evaluated using the second order statistics in (12). It is found 


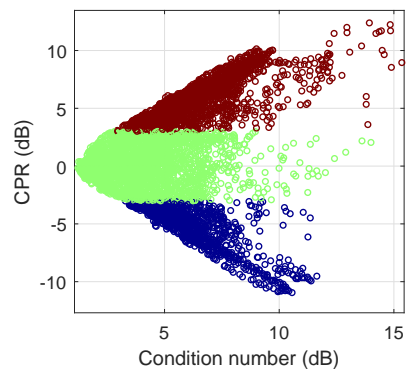

(a)

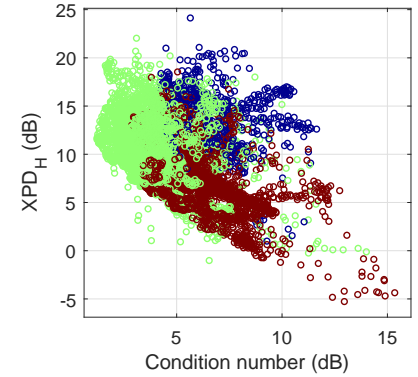

(b)
Fig. 14. Scatterplots of the condition number versus $\mathrm{CPR}$ (a) and $\mathrm{XPD}_{\mathrm{H}}$ (b).

that $\epsilon=7.58 \%$, which implies that the Kronecker model may not be suitable for this scenario. Same conclusions are found in [27]-[29].

These results appear to be in contradiction with those presented in Fig. 10, where the condition number remains relatively low throughout the tunnel. We further calculate the correlation coefficients per stationarity region and plot them versus distance in Fig. 13. Indeed, the correlation coefficients remain high throughout the tunnel and no correlation is found between them and the condition number. This suggests that the main source of the multiplexing gain (i.e. having singular values of similar power representing parallel orthogonal subchannels) is not the decorrelation of the DP subchannels, but rather the DP orthogonality or diagonalization of the channel matrix that is maintained by high XPD and CPR close to unity. Fig. 14 depicts the scatterplots of the condition number versus CPR and $\mathrm{XPD}_{\mathrm{H}}$. The plots are split into three parts according to the CPR level: $\mathrm{CPR}>3 \mathrm{~dB}$ (red), $\mathrm{CPR}<-3 \mathrm{~dB}$ (blue), and in between (green). Fig. 14 (a) shows that there is a correlation between the CPR and the condition number, especially in the high CPR regions (red and blue) where the condition number decreases as CPR approaches $0 \mathrm{~dB}$. It also shows that the condition number is lower-bounded by the magnitude of the CPR. Fig. 14 (b) shows that the condition number decreases as $\mathrm{XPD}_{\mathrm{H}}$ increases in the green and red regions. In other words, even when the power of $\mathrm{VV}$ is higher than $\mathrm{HH}$ (red), having less leakage from the $\mathrm{HH}$ (higher $\mathrm{XPD}_{\mathrm{H}}$ ) improves the channel multiplexing gain. For the red and green regions combined, the Pearson coefficient of correlation is calculated between the condition number and the magnitude of the CPR and XPD, and is found to be 0.8 and -0.6 , respectively.

\section{CONCLUSIONS}

In this paper, we present a measurement-based analysis of a non-stationary V2I DP wireless channel in a road tunnel environment. The measurements are performed at $1.35 \mathrm{GHz}$ and $90 \mathrm{~km} / \mathrm{h}$ in the Beveren tunnel in Belgium under traffic conditions. The path gain is modeled using the one-slope model. We apply the framework of the local scattering function and channel correlation function to characterize the stationarity time. The temporal evolution of the RMS delay and Doppler spreads, as well as the CPR and XPD are investigated. A lognormal model is found to best fit the statistical distribution of these parameters. Investigating the impact of antenna polarization shows that the $\mathrm{V}$ polarization is more advantageous, as it provides higher path gain and longer stationarity time by $5 \%$, in addition to smaller delay and Doppler spreads (by $19 \%$ and $18 \%$ on average, respectively) compared to the $\mathrm{H}$ polarization. As for the impact of antenna radiation pattern, a more directional antenna is found to provide a longer stationarity time by $21 \%$, as well as smaller delay and Doppler spreads (by $52 \%$ and $32 \%$ on average, respectively), thus proving to be more beneficial than a wide-angle type of antenna pattern. Moreover, the impact of normalization on the DP capacity is investigated, and a new approach is proposed that maintains the conservation of energy. The DP channel is found to have a low condition number on average $(5.5 \mathrm{~dB})$, which is good for multiplexing gain. The correlation properties are measured using the full correlation matrix, while the Kronecker model is found to provide less accurate results by $7.58 \%$. Large correlation $(>0.7)$ among DP subchannels is observed, and no correlation is found with the condition number. The condition number is found to depend on the orthogonality rather than the decorrelation of the DP subchannels, giving DP MIMO an advantage over spatial MIMO in LOS scenarios. Future work will consist of modeling the non-stationary fading process based on the LSF and CCF framework for vehicular communication channels simulation.

\section{REFERENCES}

[1] A. F. Molisch, F. Tufvesson, J. Karedal, and C. F. Mecklenbrauker, "A survey on vehicle-to-vehicle propagation channels," IEEE Wireless Communications, vol. 16, no. 6, pp. 12-22, 2009.

[2] D. G. Dudley, M. Lienard, S. F. Mahmoud, and P. Degauque, "Wireless propagation in tunnels," IEEE Antennas and Propagation Magazine, vol. 49, no. 2, pp. 11-26, 2007.

[3] A. Hrovat, G. Kandus, and T. Javornik, "A survey of radio propagation modeling for tunnels," IEEE Communications Surveys \& Tutorials, vol. 16, no. 2, pp. 658-669, 2014.

[4] M. Lienard, P. Degauque, J. Baudet, and D. Degardin, "Investigation on MIMO channels in subway tunnels," IEEE Journal on Selected Areas in Communications, vol. 21, no. 3, pp. 332-339, 2003.

[5] Z. Sun and I. F. Akyildiz, "Channel modeling and analysis for wireless networks in underground mines and road tunnels," IEEE Transactions on communications, vol. 58, no. 6, 2010.

[6] J. Molina-Garcia-Pardo, M. Lienard, and P. Degauque, "Propagation in tunnels: Experimental investigations and channel modeling in a wide frequency band for MIMO applications," EURASIP Journal on Wireless Communications and Networking, vol. 2009, p. 7, 2009.

[7] M. Liénard, S. Bétrencourt, and P. Degauque, "Propagation in road tunnels: a statistical analysis of the field distribution and impact of the traffic," in Annales des télécommunications, vol. 55, no. 11-12. Springer, 2000, pp. 623-631.

[8] F. M. Pallares, F. P. Juan, and L. Juan-Llacer, "Analysis of path loss and delay spread at $900 \mathrm{MHz}$ and $2.1 \mathrm{GHz}$ while entering tunnels," IEEE Transactions on Vehicular Technology, vol. 50, no. 3, pp. 767-776, 2001.

[9] C. Garcia-Pardo, J.-M. Molina-García-Pardo, M. Lienard, D. P. Gaillot, and P. Degauque, "Double directional channel measurements in an arched tunnel and interpretation using ray tracing in a rectangular tunnel," Progress In Electromagnetics Research, vol. 22, pp. 91-107, 2012.

[10] L. Bernadó, A. Roma, A. Paier, T. Zemen, N. Czink, J. Karedal, A. Thiel, F. Tufvesson, A. F. Molisch, and C. F. Mecklenbrauker, "Intunnel vehicular radio channel characterization," in Vehicular Technology Conference (VTC Spring), 2011 IEEE 73rd. IEEE, 2011, pp. 1-5.

[11] M. Yusuf, E. Tanghe, L. Martens, P. Laly, D. P. Gaillot, M. Linard, P. Degauque, and W. Joseph, "Experimental investigation of V2I radio channel in an arched tunnel," in 2019 13th European Conference on Antennas and Propagation (EuCAP), March 2019, pp. 1-5. 
[12] L. Bernadó, T. Zemen, F. Tufvesson, A. F. Molisch, and C. F. Mecklenbräuker, "The (in-) validity of the WSSUS assumption in vehicular radio channels." in PIMRC, 2012, pp. 1757-1762.

[13] G. Matz, "On non-WSSUS wireless fading channels," IEEE Transactions on Wireless Communications, vol. 4, no. 5, pp. 2465-2478, 2005.

[14] M. Yusuf, E. Tanghe, F. Challita, P. Laly, D. Gaillot, M. Lienard, L. Martens, and W. Joseph, "Stationarity analysis of V2I radio channel in a suburban environment," IEEE Transactions on Vehicular Technology, pp. 1-1, 2019.

[15] T. Abbas, J. Karedal, and F. Tufvesson, "Measurement-based analysis: The effect of complementary antennas and diversity on vehicle-tovehicle communication," IEEE Antennas and Wireless Propagation Letters, vol. 12, pp. 309-312, 2013.

[16] Y. He, X. Cheng, and G. L. Stuber, "On polarization channel modeling," IEEE Wireless Communications, vol. 23, no. 1, pp. 80-86, 2016.

[17] C. Guo, F. Liu, S. Chen, C. Feng, and Z. Zeng, "Advances on exploiting polarization in wireless communications: Channels, technologies, and applications," IEEE Communications Surveys Tutorials, vol. 19, no. 1, pp. 125-166, 2017.

[18] C. Oestges, B. Clerckx, M. Guillaud, and M. Debbah, "Dual-polarized wireless communications: from propagation models to system performance evaluation," IEEE Transactions on Wireless Communications, vol. 7, no. 10, pp. 4019-4031, 2008.

[19] "Technical specification group radio access network; vehicle-toeverything (V2X) services based on LTE; user equipment (UE) radio transmission and reception. TR 36.786 v14.0.0," 3GPP, Tech. Rep., 2017.

[20] A. Timm-Giel, A. P. Subramanian, K. Dhanasekaran, V. Navda, and S. R. Das, "Directional antennas for vehicular communication - experimental results," in 2007 IEEE 65th Vehicular Technology Conference VTC2007-Spring, 2007, pp. 357-361.

[21] D. Eckhoff, A. Brummer, and C. Sommer, "On the impact of antenna patterns on VANET simulation," in 2016 IEEE Vehicular Networking Conference (VNC), 2016, pp. 1-4.

[22] A. Ispas, C. Schneider, G. Ascheid, and R. Thom, "Analysis of the local quasi-stationarity of measured dual-polarized MIMO channels," IEEE Transactions on Vehicular Technology, vol. 64, no. 8, pp. 3481-3493, 2015.

[23] V. Nikolaidis, N. Moraitis, and A. G. Kanatas, "Dual-polarized narrowband MIMO LMS channel measurements in urban environments," IEEE Transactions on Antennas and Propagation, vol. 65, no. 2, pp. 763-774, 2017.

[24] P. Laly, D. P. Gaillot, M. Liénard, P. Degauque, E. Tanghe, W. Joseph, and L. Martens, "Flexible real-time MIMO channel sounder for multidimensional polarimetric parameter estimation," in Antenna Measurements \& Applications (CAMA), 2015 IEEE Conference on. IEEE, 2015, pp. $1-3$.

[25] P. Kyritsi, D. C. Cox, R. A. Valenzuela, and P. W. Wolniansky, "Effect of antenna polarization on the capacity of a multiple element system in an indoor environment," IEEE Journal on Selected Areas in Communications, vol. 20, no. 6, pp. 1227-1239, 2002.

[26] J. W. Wallace, M. A. Jensen, A. L. Swindlehurst, and B. D. Jeffs, "Experimental characterization of the MIMO wireless channel: data acquisition and analysis," IEEE Transactions on Wireless Communications, vol. 2, no. 2, pp. 335-343, 2003.

[27] C. Oestges, V. Erceg, and A. J. Paulraj, "Propagation modeling of MIMO multipolarized fixed wireless channels," IEEE Transactions on Vehicular Technology, vol. 53, no. 3, pp. 644-654, 2004.

[28] Da-Shan Shiu, G. J. Foschini, M. J. Gans, and J. M. Kahn, "Fading correlation and its effect on the capacity of multielement antenna systems," IEEE Transactions on Communications, vol. 48, no. 3, pp. 502-513, 2000.

[29] J. P. Kermoal, L. Schumacher, F. Frederiksen, and P. E. Mogensen, "Polarization diversity in MIMO radio channels: experimental validation of a stochastic model and performance assessment," in IEEE 54th Vehicular Technology Conference. VTC Fall 2001. Proceedings (Cat. No.01CH37211), vol. 1, 2001, pp. 22-26 vol.1.

[30] Kai Yu, M. Bengtsson, B. Ottersten, D. McNamara, P. Karlsson, and M. Beach, "Second order statistics of NLOS indoor MIMO channels based on $5.2 \mathrm{GHz}$ measurements," in GLOBECOM'01. IEEE Global Telecommunications Conference (Cat. No.01CH37270), vol. 1, 2001, pp. $156-160$ vol.1.

[31] S. Cheng, D. P. Gaillot, E. Tanghe, P. Laly, T. Demol, W. Joseph, L. Martens, and M. Linard, "Polarimetric distance-dependent models for large hall scenarios," IEEE Transactions on Antennas and Propagation, vol. 64, no. 5, pp. 1907-1917, 2016.

[32] J. M. Molina-Garcia-Pardo, M. Lienard, P. Degauque, D. G. Dudley, and L. Juan-Llacer, "Interpretation of MIMO channel characteristics in rectangular tunnels from modal theory," IEEE Transactions on Vehicular Technology, vol. 57, no. 3, pp. 1974-1979, 2008.

[33] J. M. Molina-Garcia-Pardo, M. Lienard, A. Nasr, and P. Degauque, "On the possibility of interpreting field variations and polarization in arched tunnels using a model for propagation in rectangular or circular tunnels," IEEE Transactions on Antennas and Propagation, vol. 56, no. 4, pp. 1206-1211, 2008.

[34] M. H. Kermani and M. Kamarei, "A ray-tracing method for predicting delay spread in tunnel environments," in 2000 IEEE International Conference on Personal Wireless Communications. Conference Proceedings (Cat. No.00TH8488), 2000, pp. 538-542.

[35] S. Bashir, "Effect of antenna position and polarization on UWB propagation channel in underground mines and tunnels," IEEE Transactions on Antennas and Propagation, vol. 62, no. 9, pp. 4771-4779, 2014.

[36] J. Molina-Garcia-Pardo, M. Lienard, P. Degauque, C. Garcia-Pardo, and L. Juan-Llacer, "MIMO channel capacity with polarization diversity in arched tunnels," IEEE Antennas and Wireless Propagation Letters, vol. 8, pp. 1186-1189, 2009.

[37] D. McNamara, C. Pistorius, and J. Malherbe, "The uniform geometrical theory of diffraction," Artech House, London, 1990.

[38] J. W. Wallace, M. A. Jensen, A. Gummalla, and H. B. Lee, "Experimental characterization of the outdoor MIMO wireless channel temporal variation," IEEE Transactions on Vehicular Technology, vol. 56, no. 3, pp. 1041-1049, 2007.

[39] H. W. Lilliefors, "On the kolmogorov-smirnov test for normality with mean and variance unknown," Journal of the American statistical Association, vol. 62, no. 318, pp. 399-402, 1967.

[40] V. R. Anreddy and M. A. Ingram, "Capacity of measured ricean and rayleigh indoor MIMO channels at $2.4 \mathrm{GHz}$ with polarization and spatial diversity," in IEEE Wireless Communications and Networking Conference, 2006. WCNC 2006., vol. 2, 2006, pp. 946-951.

[41] S. Schindler and H. Mellein, "Assessing a MIMO channel," Rohde \& Schwarz white paper, Tech. Rep., 2011.

[42] "MIMO performance and condition number in LTE test," Agilent Technologies Application Note, Tech. Rep., 2009. 